\title{
Biological Variation of Cardiac Troponins in Chronic Kidney Disease
}

Jones RA, ${ }^{1}$ Barratt J, ${ }^{2}$ Brettell EA, ${ }^{3}$ Cockwell P, ${ }^{4}$ Dalton RN, ${ }^{5}$ Deeks JJ ${ }^{3,}, 6,7$ Eaglestone G, Pellatt-Higgins T, ${ }^{9}$ Kalra PA, ${ }^{10}$ Khunti K, ${ }^{11}$ Morris FS, ${ }^{8}$ Ottridge RS, ${ }^{3}$ Sitch AJ, ${ }^{6,7}$ Stevens PE ${ }^{8}$ Sharpe CC, ${ }^{12}$ Sutton AJ, ${ }^{13}$ Taal MW, ${ }^{14}$ Lamb EJ, ${ }^{* * *}$ on behalf of the eGFR-C study group.

***orresponding author

${ }^{1}$ Clinical Biochemistry, East Kent Hospitals University NHS Foundation Trust, Canterbury, Kent, CT1 3NG, UK, ${ }^{2}$ University Hospitals of Leicester, ${ }^{3}$ Birmingham Clinical Trials Unit, Institute of Applied Health Research, University of Birmingham, Birmingham B15 2TT UK, ${ }^{4}$ Renal Medicine, Queen Elizabeth Hospital Birmingham and Institute of Inflammation and Ageing, University of Birmingham, Birmingham B15 2TT UK, ${ }^{5}$ Evelina London Children's Hospital, London SE1 7EH, ${ }^{6}$ Test Evaluation Research Group, University of Birmingham, Birmingham B15 2TT, UK, ${ }^{7}$ NIHR Birmingham Biomedical Research Centre, University of Birmingham and University Hospitals Birmingham NHS Foundation Trust, B15 2TT, UK ${ }^{8}$ Kent Kidney Care Centre, East Kent Hospitals University NHS Foundation Trust, Canterbury, Kent, CT1 3NG, UK, ${ }^{9}$ Centre for Health Services Studies, University of Kent, Canterbury, CT2 7NF, UK, ${ }^{10}$ Salford Royal NHS Foundation Trust, Salford, M6 8HD, UK ${ }^{11}$ University of Leicester, ${ }^{12}$ King's College London \& King's College Hospital NHS Foundation Trust, London, SE5 9RJ, ${ }^{13}$ Institute of Health Economics, Edmonton, Canada, T5J 3N4, ${ }^{14}$ Division of Medical Sciences and Graduate Entry Medicine, University of Nottingham, Royal Derby Hospital, Uttoxeter Road, Derby, DE22 3DT, UK.

Address correspondence to: Dr Edmund Lamb, Consultant Clinical Scientist, Clinical Biochemistry, East Kent Hospitals University NHS Foundation Trust, Kent and Canterbury Hospital, Canterbury, Kent, UK, CT1 3NG. Telephone: 01227 864112, Facsimile: 01227 783077, E-mail: elamb@nhs.net

Word count (excluding abstract, references, etc): 2989

Word count of abstract: 277 


\section{Abstract}

Patients with chronic kidney disease (CKD) often have increased plasma cardiac troponin (cTn) concentration in the absence of myocardial infarction (MI). Incidence of MI is high in this population and diagnosis, particularly of non ST-segment elevation MI (NSTEMI), is challenging. Knowledge of biological variation aids understanding of serial cTn measurements and could improve interpretation in clinical practice. The National Academy of Clinical Biochemistry (NACB) recommended the use of a $20 \%$ reference change value $(\mathrm{RCV})$ in patients with kidney failure. The aim of this study was to calculate the biological variation of cardiac troponin $\mathrm{I}(\mathrm{cTnI})$ and $\mathrm{T}(\mathrm{cTnT})$ in patients with moderate $\mathrm{CKD}$ (glomerular filtration rate [GFR] $30-59 \mathrm{~mL} / \mathrm{min} / 1.73 \mathrm{~m}^{2}$ ).

Plasma samples were obtained from twenty patients (median GFR $43.0 \mathrm{~mL} / \mathrm{min} / 1.73 \mathrm{~m}^{2}$ )

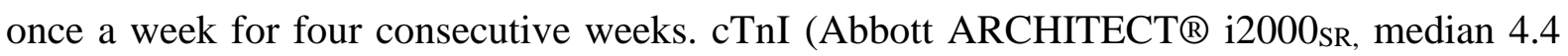
$\mathrm{ng} / \mathrm{L}$, upper $99^{\text {th }}$ percentile of reference population $26.2 \mathrm{ng} / \mathrm{L}$ ) and cTnT (Roche Cobas ${ }^{\circledR}$ e601, median $11.7 \mathrm{ng} / \mathrm{L}$, upper $99^{\text {th }}$ percentile of reference population $14 \mathrm{ng} / \mathrm{L}$ ) were measured in duplicate using high sensitivity assays. After outlier removal and log transformation, eighteen patients' data were subject to ANOVA and within-subject $\left(\mathrm{CV}_{\mathrm{I}}\right)$, between-subject $\left(\mathrm{CV}_{\mathrm{G}}\right)$ and analytical $\left(\mathrm{CV}_{\mathrm{A}}\right)$ variation calculated. Variation for cTnI was $15.0 \%, 105.6 \%, 8.3 \%$ respectively and for cTnT $7.4 \%, 78.4 \%, 3.1 \%$, respectively. RCVs for increasing and decreasing troponin concentrations were $+60 \% /-38 \%$ for cTnI and $+25 \% /$ $20 \%$ for cTnT.

The observed RCV for cTnT is broadly compatible with the NACB recommendation, but for cTnI larger changes are required to define significant change. The incorporation of separate RCVs for cTnI and cTnT, and separate RCVs for rising and falling concentrations of cardiac troponin, should be considered when developing guidance for interpretation of sequential cardiac troponin measurements. 


\section{Introduction}

Chronic kidney disease (CKD) is common: $4.5 \%$ of the UK population have a glomerular filtration rate (GFR) below $60 \mathrm{~mL} / \mathrm{min} / 1.73 \mathrm{~m}^{2}$. ${ }^{1}$ The majority of individuals with CKD have moderate disease (CKD category G3) defined by a GFR of 30 to $59 \mathrm{~mL} / \mathrm{min} / 1.73 \mathrm{~m}^{2}$. People with CKD have an increased prevalence of cardiovascular disease ${ }^{2,3}$ and an increased cardiovascular mortality risk with declining renal function. ${ }^{4-8}$ Consequently, patients with moderate $\mathrm{CKD}$ are more likely to die from cardiovascular complications than progress to renal failure requiring renal replacement therapy.

Diagnosis of acute myocardial infarction requires: (a) evidence of myocardial injury by observing at least one measure of cardiac troponin concentration exceeding the upper $99^{\text {th }}$ percentile of a healthy reference population; (b) confirmation that such injury is acute, through observing a rise and/or fall of cardiac troponin concentration and; (c) evidence of myocardial ischaemia as demonstrated by accompanying characteristic symptoms and signs of myocardial infarction (e.g. electrocardiogram changes). ${ }^{9}$ Patients with CKD are frequently observed to have concentrations of cardiac troponin that exceed the $99^{\text {th }}$ percentile in the absence of myocardial infarction. ${ }^{9-12}$ The increased plasma troponin concentration observed in CKD is most likely a reflection of the multifaceted burden of cardiovascular disease and creates a significant diagnostic problem. ${ }^{9,10,13-15}$ The diagnosis of myocardial infarction in patients with CKD can be further complicated by absence of ischaemic symptoms and characteristic electrocardiogram changes. ${ }^{13,16-18}$ In this situation, observation of changing cardiac troponin concentration over time is a useful pointer towards myocardial infarction, although acute volume overload or heart failure may also cause this pattern. ${ }^{9}$

There is little published data relating to the biological variation of cardiac troponin in patients with moderate CKD. Knowledge of the biological (and analytical) variation can be used to calculate reference change values (RCVs), which can be used to determine whether a change in serial measures of a biomarker are statistically significant. ${ }^{19}$ This is pertinent to the diagnosis of acute coronary syndromes since other medical conditions, including CKD, increase cardiac troponin concentrations in the absence of myocardial infarction. Defining significant change in cardiac troponin concentration is an area of active debate. In 2007, the 
National Academy of Clinical Biochemistry (NACB) suggested a change of $20 \%$ should be considered significant amongst dialysis patients, although this recommendation predated use of high sensitivity cardiac troponin assays and did not consider biological variation. ${ }^{20}$ The recent Fourth Universal Definition of Myocardial Infarction considers the difficulties of defining significant change. Whilst a troponin concentration change $>50 \%$ has been considered to exceed that which could be attributed to biological and analytical variability, smaller changes $(>20 \%)$ are significant when the initial baseline value exceeds the $99^{\text {th }}$ percentile of the reference population. Furthermore, there is a move towards defining change in absolute (concentration) rather than relative (percentage) terms, although it is acknowledged that such values will be assay dependent. ${ }^{9,21}$ Generally, definitions of significant change have not discriminated between cardiac troponin $\mathrm{T}$ (cTnT) and cardiac troponin I (cTnI). The aim of the present study was to establish the total variation $\left(\mathrm{CV}_{\mathrm{T}}\right)$, within-subject biological variation $\left(\mathrm{CV}_{\mathrm{I}}\right)$ and analytical variation $\left(\mathrm{CV}_{\mathrm{A}}\right)$ of $\mathrm{cTnT}$ and $\mathrm{cTnI}$ using high-sensitivity assays in patients with clinically stable moderate CKD in order to derive RCVs.

\section{Study Participants and Methods}

Patients were recruited from nephrology clinics at the Kent Kidney Care Centre, Canterbury, UK, between August 2014 and July 2015. Twenty white participants with moderate CKD were invited to participate in the biological variability sub-study of the multi-centre UK prospective longitudinal eGFR-C study. ${ }^{22}$ Inclusion criteria comprised an estimated GFR between 30 and $59 \mathrm{~mL} / \mathrm{min} / 1.73 \mathrm{~m}^{2}$ sustained over at least 90 days prior to the study. GFR was estimated using the Modification of Diet in Renal Disease (MDRD) Study equation ${ }^{23}$ and an enzymatic creatinine assay (Abbott Laboratories) standardised to the reference materials NIST SRM 967 and 914. Exclusion criteria were age <18 years, pregnancy, breastfeeding, an episode of acute kidney injury within the last six months, known current alcohol or drug abuse, amputee (whole or part-limb), kidney transplant recipient and cognitive impairment. Patients provided written informed consent. The study had ethical approval (South-East Coast Research Ethics Committee, reference 11/LO/1304). The study conforms to the internationally agreed checklist for the reporting of studies of biological variation. ${ }^{24}$ 
Blood was collected into ethylenediaminetetraacetic acid (EDTA) (Vacuette ${ }^{\circledR}$, Greiner BioOne International) tubes using standard venepuncture procedures. Samples were obtained once a week for four consecutive weeks with standardisation to the same time of day (morning) and day of the week to minimize pre-analytical variables. Samples were centrifuged within 6 hours of venepuncture and plasma pipetted into $2 \mathrm{~mL}$ microtubes (Sarstedt). Plasma was stored at $-80^{\circ} \mathrm{C}$ for approximately 24 months before cardiac troponins were measured in a single batch.

Cardiac troponins were measured in duplicate using two high-sensitivity assays. On the morning of analysis, samples were thawed at room temperature and mixed by inversion. Immediately prior to analysis, samples were centrifuged at 3,300 $g$ for 10 minutes. Plasma cTnI was measured using the ARCHITECT® STAT high-sensitivity cTnI chemiluminescent microparticle immunoassay (Abbott Laboratories, Chicago, Illinois, USA, product code $3 \mathrm{P} 25)$ assay ( $99^{\text {th }}$ percentile of a healthy reference population: female $(15.6 \mathrm{ng} / \mathrm{L})$, male $(34.2$ ng/L), combined (26.2 ng/L)) on the Abbott ARCHITECT® i2000 ${ }_{\text {SR }}$ module. Plasma cTnT was measured using the Elecsys ${ }^{\circledR}$ high-sensitivity cTnT electrochemiluminescent sandwich immunoassay on the Roche Cobas ${ }^{\circledR}$ e601 (Roche Diagnostics, Risch-Rotkreuz, Switzerland) analyser platform ( $99^{\text {th }}$ percentile of a healthy reference population $14 \mathrm{ng} / \mathrm{L}$ ). The measurement of both cardiac troponins was undertaken on the same day by a single operator using one instrument that had been calibrated using a single batch of standards, reagents and quality controls. The samples for each patient were analysed in duplicate in random order to minimise any effects of assay drift.

\section{Data analysis}

Samples with measures below the limit of detection for either the cTnI assay $(\leq 1.2 \mathrm{ng} / \mathrm{L})$ or the cTnT assay $(\leq 5 \mathrm{ng} / \mathrm{L})$ were excluded. Normality was assessed using the Shapiro-Wilk test (Analyse-it Software Ltd., Leeds, UK) which showed a non-Gaussian distribution. The data were therefore log-transformed using a natural logarithm as recommended. ${ }^{24,25}$ For both cardiac troponins, Cochran's test identified two values that differed markedly between duplicate measures and one value that differed markedly between the series of replicate measures; these were therefore removed. Where one duplicate of a sample was excluded (or absent), both duplicate results were excluded from statistical analysis. ${ }^{26}$ The dataset of each patient was then subject to outlier removal by Reed's test; no subjects were excluded based on this test. The dataset belonging to any patient with less than three (out of four) duplicate 
cardiac troponin measures available were completely excluded from statistical analysis. On this basis, a total of three patients were excluded; one individual was excluded from all analysis; one individual was excluded from cTnI analysis only and one individual was excluded from cTnT analysis only. Details of excluded measures are shown in supplementary table S1. The log-transformed data from 18 patients were subject to ANOVA (Minitab Statistical Software, Minitab Ltd, Coventry, UK) and estimates of biological variation were generated for $\mathrm{CV}_{\mathrm{T}}, \mathrm{CV}_{\mathrm{A}}$ and $\mathrm{CV}_{\mathrm{I}}$. Data was then back-transformed allowing derivation of the exact $\mathrm{CV}$ values $\left[\left[\sqrt{\exp \left(\mathrm{S}^{2}\right)-1} \times 100\right] .{ }^{27}\right.$ Power $(>80 \%)^{28}$ and width of confidence intervals $(\mathrm{CI})$ for the $\mathrm{CV}_{\mathrm{I}}{ }^{29}$ were estimated according to standard methods. Calculation of bidirectional (positive and negative) RCV's was appropriate for the definition of acute myocardial infarction (a rise and/or fall in cardiac troponin). Furthermore, since it cannot be assumed that the positive RCV (rise) is the same as the negative RCV (fall), asymmetrical (log-normal) RCV's were calculated using the approach for log-normal data proposed by Fokkema. $^{25}$

\section{Results}

Characteristics of the study participants are shown in Table 1 . Three patients had type 2 diabetes mellitus, seven ischaemic heart disease, one angina and two had heart failure. One participant was a current smoker and 10 were ex-smokers. Antihypertensive and cholesterolreducing drugs were being taken by 15 and 13 patients, respectively. Seven of the participants had urine albumin concentrations between 3 and $30 \mathrm{mg} / \mathrm{mmol}$ creatinine, and a further four had urine albumin concentrations $>30 \mathrm{mg} / \mathrm{mmol}$. No participants had a significant change in kidney function during the study nor an acute cardiac event. ${ }^{30}$

The median concentration overall (all patients, all study points) of both cardiac troponins was below the relative $99^{\text {th }}$ percentile derived from a healthy reference population. For cTnT the median cTnT concentration of seven patients exceeded the $99^{\text {th }}$ percentile (Fig 1.A) whereas for cTnI, no individual patient had a median concentration over all study points exceeding the $99^{\text {th }}$ percentile (Fig 1.B). The highest median concentration of cTnT observed in an individual was $37.6 \mathrm{ng} / \mathrm{L}$ whereas the highest median value for an individual for cTnI was $13.7 \mathrm{ng} / \mathrm{L}$. 
Biological and analytical variation data are shown in Table 2. The within-subject and analytical variability of cTnT was approximately half that of cTnI. Consequently, the positive and negative RCV's were lower for cTnT (25\%/-20\%) than for cTnI (60\%/-38\%). 


\section{Discussion}

Defining a statistically significant change in serial troponin concentrations should be established from biological variability studies. ${ }^{31}$ There is a literature describing the biological variation of cardiac troponins in patients with kidney failure treated by dialysis but, to the best of our knowledge, this is the first study to report the biological variation of both cTnI and cTnT measured using high-sensitivity assays in patients with moderate CKD. We observed within-subject biological variation of $7.4 \%$ (CI $5.6 \%$ to $9.2 \%$ ) and $15.0 \%$ (CI $11.3 \%$ to $18.7 \%$ ) for $\mathrm{cTnT}$ and $\mathrm{cTnI}$, respectively. RCVs for increasing and decreasing troponin concentrations were $+25 \% /-20 \%$ for $\mathrm{cTnT}$ and $+60 \% /-38 \%$ for $\mathrm{cTnI}$.

The estimates of within-subject biological variation for cardiac troponin reported in the present study are similar to those previously reported in patients undergoing haemodialysis. For example, Fahim et al reported a $\mathrm{CV}_{\mathrm{I}}$ for $\mathrm{cTnT}$ of $7.9 \%$ in dialysis patients. ${ }^{32}$ Among studies that simultaneously measured both $\mathrm{cTnI}$ and $\mathrm{cTnT},{ }^{33,34}$ including the present study, the within-subject variability has been consistently lower for cTnT than for cTnI. In patients with renal failure undergoing haemodialysis treatment Mbagaya et al reported slightly higher $\mathrm{CV}_{\mathrm{I}}$ values compared to the present study for cTnT and cTnI of $10.5 \%$ and $20.2 \%$ respectively. ${ }^{34}$ Aakre et al estimated the $\mathrm{CV}_{\mathrm{I}}$ of cardiac troponins amongst stable haemodialysis patients and reported values of $8.3 \%$ and $15.6 \%$ for cTnT and cTnI respectively. In the same study $\mathrm{CV}_{\mathrm{I}}$ values amongst healthy individuals of $8.3 \%$ (cTnT) and $14.3 \%$ (cTnI) were reported. ${ }^{33}$ Corte et al observed within-subject biological variation for cTnT of $14.7 \%$ amongst patients with kidney failure compared to 5.9\% amongst healthy individuals, although the study design was somewhat different between the two groups. ${ }^{35}$ Amongst patients with CKD not receiving dialysis (median estimated GFR $17 \mathrm{~mL} / \mathrm{min} / 1.73$ $\mathrm{m}^{2}$ ), within-day biological variability for cTnI of $8 \%$ to $9 \%$ has been reported. ${ }^{36}$ Overall, reported variability appears comparable between studies including haemodialysis patients, CKD patients and healthy individuals.

When considering any change in a patient's results, healthcare practitioners need to be able to distinguish true change ('signal') from the 'noise' of variability. In clinical practice, biological variation is best considered in terms of the RCV, which takes both biological and analytical variation into account. RCV's for cTnT in the present study (25\%/-20\%) are generally comparable to those previously reported in dialysis patients: $33 \% /-25 \%,{ }^{32} 26 \% /-$ 
$21 \%{ }^{33}$ and $34 \% /-26 \% .{ }^{34}$ The RCV's for cTnT reported in the present study are also broadly compatible with the NACB's definition of a significant change of $20 \% .^{20}$

For cTnI, however, our data suggests a larger difference (60\%/-38\%) is required to define significant change. Similarly, reported RCV's for cTnI amongst dialysis patients were 53\%/$35 \%{ }^{33}$ and $80 \% /-44 \%,{ }^{34}$ and amongst CKD patients not receiving dialysis $34 \% /-26 \% .{ }^{36}$ The reported positive and negative RCV values therefore consistently exceed the $20 \%$ critical difference recommended by the NACB, in some cases by several fold, and would stretch the critical relative differences discussed within the Fourth Universal Definition of Myocardial Infarction. ${ }^{9,20}$ Use of a $20 \%$ critical difference to interpret serial measures of cTnI could contribute to misleading diagnoses of acute myocardial infarction in patients with moderate CKD. It is also clear that the data does not have a symmetric distribution, i.e. the same critical difference cannot be applied to rising and falling troponin concentrations. Our data and that of others, ${ }^{33,34,36}$ particularly for cTnI, demonstrate that the biological variation of troponin concentration is non-Gaussian and that RCVs will differ between rising and falling concentrations. Similarly, should absolute, as opposed to relative, delta values be used to detect change, as has been suggested, the same issue will pertain regarding different rising and falling troponin concentrations. ${ }^{9}$

As previously reported, particularly amongst dialysis patients, ${ }^{32-34}$ but also in earlier studies of non-dialysis CKD patients, ${ }^{10,11}$ cTnT is more commonly increased above the $99^{\text {th }}$ percentile than cTnI. The reason for this is not the main focus of the present study but various explanations have been proposed including different cTnI and cTnT release kinetics or differences in clearance from the circulation. This observation is reinforced by the present study with concentrations of cTnT commonly exceeding the $99^{\text {th }}$ percentile reference interval whilst cTnI concentrations were predominantly normal.

The study was adequately powered based on essential elements of the study design (number of individuals, samples and replicates). ${ }^{28}$ In relation to analytical performance specifications defined by the ratio of $\mathrm{CV}_{\mathrm{A}}$ to $\mathrm{CV}_{\mathrm{I}}$, the cTnT (0.4) and cTnI (0.5) assays met desirable and minimum quality specifications respectively. ${ }^{26}$ The study followed a strict design to minimise pre-analytical and analytical variation and investigator bias. ${ }^{24}$ Outliers in the data were excluded using a formal exclusion protocol and a strength of this study is that few data were excluded prior to ANOVA. Estimation of components of variation was derived using a nested 
ANOVA approach, which takes into account analytical variation for estimation of withinsubject biological variation. The participants were derived from a patient group which is a major population in which detection of myocardial infarction is a clinically relevant issue. Participants had stable kidney disease, suggesting that the variation we have reported is physiological and not pathological in nature. The methods used to measure cTnT and cTnI met criteria for the definition of high-sensitivity assays ${ }^{31,37}$ and are recommended by the National Institute for Health and Care Excellence (NICE) for the early rule out of myocardial infarction. ${ }^{38}$

Our study has some limitations. The cohort studied was recruited from a single centre and was exclusively Caucasian: biological variability estimates may therefore not be transferable to other ethnic groups. Our study comprised participants with moderate CKD and the results may not be transferable to more advanced kidney disease. However, as discussed above, comparison with other studies of patients receiving dialysis for kidney failure suggests similar levels of biological variability. ${ }^{32-34}$ The RCV is a value derived under idealised conditions which are often not replicated in typical clinical practice (e.g. multiple operators and batches of reagents will increase the value of $C_{\mathrm{A}}$ and hence increase the RCV). Furthermore, sampling time was standardised to some extent in our study. In the clinical setting change in cTnT concentrations will also be influenced by the known circadian variation, with peak concentrations occurring in the early morning and a nadir in the evening. ${ }^{39,40}$ RCVs should therefore be considered a minimum clinically significant change. Individual laboratories may need to adjust RCVs to take into account the analytical variation of their own troponin assay. However, the values generated in this study are useful to guide understanding of change in cardiac troponin concentration in patients among whom interpretation can often be challenging. Although the recommended approach, the outlier removal process may inadvertently remove values representing true biological variation, albeit, as noted above, relatively few outliers were removed in this study. Myocardial infarction was not a measured outcome in this cohort of CKD patients. However, in a renal dialysis population, Aakre et al demonstrated successful use of the RCV to identify patients with acute cardiac events. ${ }^{33}$ Serial changes in concentration of cTnT exceeded the RCV in all four patients who experienced cardiac events and in three of four patients for cTnI. ${ }^{33}$ Finally, our samples were stored for up to 2 years at $-80^{\circ} \mathrm{C}$ prior to analysis. We are unaware of published data supporting storage over this period. However, Egger et al report good stability over one year at $-80^{\circ} \mathrm{C}$ for both cTnI and cTnT in EDTA plasma, including through two 
freeze-thaw cycles, measured using the Abbott and Roche assays respectively, suggesting good stability of these markers. ${ }^{41}$

A further consideration is the timescale over which variability is studied to generate the RCV. We have used samples obtained at weekly intervals. Variability over shorter periods of time may be more relevant when addressing the issue of diagnosis of myocardial infarction. Amongst healthy volunteers, for both cTnT and cTnI, within-subject biological variability does seem to be higher when studies are conducted over periods of days/weeks compared to studies of within-day (hourly) variability. ${ }^{33,42,43}$ Literature on this point is less clear amongst patients with kidney disease. Aakre et al studied variability in haemodialysis patients and observed lower within-subject variability when troponins were studied at 90 minute intervals over six hours (cTnT 1.9\% and cTnI 3.3\%). ${ }^{33}$ Conversely, estimates of $\mathrm{CV}_{\mathrm{I}}$ for cTnI obtained by van der Linden et al from hourly measurements over $24 \mathrm{~h}$ in patients with and without CKD were $8.7 \%$ and $9.4 \%$ respectively, closer to the results we report in the present study. ${ }^{36}$ These authors observed that the $\mathrm{CV}_{\mathrm{I}}$ did not differ significantly irrespective of whether hourly, 3-hourly, or 6-hourly sampling intervals were considered. ${ }^{36}$

In conclusion, we describe the biological variability of cTnT and cTnI in a carefully designed study using high sensitivity assays in patients with moderate CKD. Our data suggests that independent RCV's for cTnI and cTnT should be incorporated into guidance for the interpretation of cardiac troponin in patients with CKD. Furthermore, we recommend that separate positive and negative RCV's should be considered, particularly for cTnI. Whilst not the focus of our study, the same considerations would apply to the use of absolute concentration changes. Research should continue to elucidate reasons for the observed difference in prevalence of increased concentrations of cTnT compared to $\mathrm{cTnI}$ in relation to the respective $99^{\text {th }}$ percentile of a healthy reference population, and of the difference in biological variability between the two troponins, which cannot currently be explained.

\section{Acknowledgements}

We are grateful to pathology staff at the Pembury Hospital, Pembury, Kent UK for assistance with the cTnT measurements. AS and JJD are supported by the NIHR Birmingham Biomedical Research Centre at the University Hospitals Birmingham NHS Foundation Trust and the University of Birmingham. 
We acknowledge Birmingham Clinical Trials Unit for trial coordination and data management, and the Research Governance teams at the University of Birmingham and East Kent University Hospitals NHS Foundation Trust for research governance and Sponsor duties. We acknowledge the support of the National Institute of Health Research Clinical Research Network (NIHR CRN).

The eGFR-C study is funded by the National Institute for Health Research (NIHR) Health Technology Assessment Programme 11/13/01. The views expressed are those of the authors and not necessarily those of the NIHR or the Department of Health and Social Care. 


\section{References}

1. Carter JL, Stevens PE, Irving JE, et al. Estimating glomerular filtration rate: comparison of the CKD-EPI and MDRD equations in a large UK cohort with particular emphasis on the effect of age. QJM 2011;104:839-47.

2. Sarnak MJ, Levey AS, Schoolwerth AC, et al. Kidney disease as a risk factor for development of cardiovascular disease: a statement from the American Heart Association Councils on Kidney in Cardiovascular Disease, High Blood Pressure Research, Clinical Cardiology, and Epidemiology and Prevention. Circulation 2003;108:2154-69.

3. Drueke TB, Massy ZA. Atherosclerosis in CKD: differences from the general population. Nat Rev Nephrol 2010;6:723-35.

4. Wheeler DC, Townend JN, Landray MJ. Cardiovascular risk factors in predialysis patients: baseline data from the Chronic Renal Impairment in Birmingham (CRIB) study. Kidney Int Suppl 2003:S201-3.

5. Thompson S, James M, Wiebe N, et al. Cause of death in patients with reduced kidney function. J Am Soc Nephrol 2015;26:2504-11.

6. Baigent C, Burbury K, Wheeler D. Premature cardiovascular disease in chronic renal failure. Lancet 2000;356:147-52.

7. Matsushita K, van der Velde M, Astor BC, et al. Association of estimated glomerular filtration rate and albuminuria with all-cause and cardiovascular mortality in general population cohorts: a collaborative meta-analysis. Lancet 2010;375:2073-81.

8. Tonelli M, Muntner P, Lloyd A, et al. Risk of coronary events in people with chronic kidney disease compared with those with diabetes: a population-level cohort study. Lancet 2012;380:807-14.

9. Thygesen K, Alpert JS, Jaffe AS, et al. Fourth universal definition of myocardial infarction (2018). Eur Heart J 2019;40:237-69.

10. Abbas NA, John RI, Webb MC, et al. Cardiac troponins and renal function in nondialysis patients with chronic kidney disease. Clin Chem 2005;51:2059-66.

11. Lamb EJ, Kenny C, Abbas NA, et al. Cardiac troponin I concentration is commonly increased in nondialysis patients with CKD: experience with a sensitive assay. Am J Kidney Dis 2007;49:507-16.

12. Lamb EJ, Webb MC, Abbas NA. The significance of serum troponin T in patients with kidney disease: a review of the literature. Ann Clin Biochem 2004;41:1-9.

13. Gansevoort RT, Correa-Rotter R, Hemmelgarn BR, et al. Chronic kidney disease and cardiovascular risk: epidemiology, mechanisms, and prevention. Lancet 2013;382:339-52. 
14. Parikh RH, Seliger SL, deFilippi CR. Use and interpretation of high sensitivity cardiac troponins in patients with chronic kidney disease with and without acute myocardial infarction. Clin Biochem 2015;48:247-53.

15. Eggers KM, Lindahl B, Carrero JJ, et al. Cardiac troponins and their prognostic importance in patients with suspected acute coronary syndrome and renal dysfunction. Clin Chem 2017;63:1409-17.

16. Roffi M, Patrono C, Collet JP, et al. 2015 ESC Guidelines for the management of acute coronary syndromes in patients presenting without persistent ST-segment elevation: Task Force for the Management of Acute Coronary Syndromes in Patients Presenting without Persistent ST-Segment Elevation of the European Society of Cardiology (ESC). Eur Heart J 2016;37:267-315.

17. Saaby L, Poulsen TS, Hosbond S, et al. Classification of myocardial infarction: frequency and features of type 2 myocardial infarction. Am J Med 2013;126:789-97.

18. Twerenbold R, Wildi K, Jaeger C, et al. Optimal cutoff levels of more sensitive cardiac troponin assays for the early diagnosis of myocardial infarction in patients with renal dysfunction. Circulation 2015;131:2041-50.

19. Fraser CG. Making better use of differences in serial laboratory results. Ann Clin Biochem 2012;49:1-3.

20. National Academy of Clinical Biochemistry. National Academy of Clinical Biochemistry laboratory medicine practice guidelines: use of cardiac troponin and B-type natriuretic peptide or $\mathrm{N}$-terminal proB-type natriuretic peptide for etiologies other than acute coronary syndromes and heart failure. Clin Chem 2007;53:2086-96.

21. Mueller M, Biener M, Vafaie M, et al. Absolute and relative kinetic changes of highsensitivity cardiac troponin $\mathrm{T}$ in acute coronary syndrome and in patients with increased troponin in the absence of acute coronary syndrome. Clin Chem 2012;58:209-18.

22. Lamb EJ, Brettell EA, Cockwell P, et al. The eGFR-C study: accuracy of glomerular filtration rate (GFR) estimation using creatinine and cystatin $\mathrm{C}$ and albuminuria for monitoring disease progression in patients with stage 3 chronic kidney disease--prospective longitudinal study in a multiethnic population. BMC Nephrol 2014;15:13.

23. Levey AS, Coresh J, Greene T, et al. Expressing the Modification of Diet in Renal Disease Study equation for estimating glomerular filtration rate with standardized serum creatinine values. Clin Chem 2007;53:766-72.

24. Bartlett WA, Braga F, Carobene A, et al. A checklist for critical appraisal of studies of biological variation. Clin Chem Lab Med 2015;53:879-85.

25. Fokkema MR, Herrmann Z, Muskiet FA, et al. Reference change values for brain natriuretic peptides revisited. Clin Chem 2006;52:1602-3. 
26. Fraser CG, Harris EK. Generation and application of data on biological variation in clinical chemistry. Crit Rev Clin Lab Sci 1989;27:409-37.

27. Cole TJ. Sympercents: symmetric percentage differences on the $100 \log (\mathrm{e})$ scale simplify the presentation of log transformed data. Statistics in Medicine 2000;19:3109-25.

28. Roraas T, Petersen PH, Sandberg S. Confidence intervals and power calculations for within-person biological variation: effect of analytical imprecision, number of replicates, number of samples, and number of individuals. Clin Chem 2012;58:1306-13.

29. Burdick RK, Graybill FA. Confidence Intervals on Variance Components: Taylor \& Francis; 1992.

30. Rowe C, Sitch AJ, Barratt J, et al. Biological variation of measured and estimated glomerular filtration rate in patients with chronic kidney disease. Kidney Int 2019;96:429-35.

31. Wu AHB, Christenson RH, Greene DN, et al. Clinical laboratory practice recommendations for the use of cardiac troponin in acute coronary syndrome: expert opinion from the Academy of the American Association for Clinical Chemistry and the Task Force on Clinical Applications of Cardiac Bio-Markers of the International Federation of Clinical Chemistry and Laboratory Medicine. Clin Chem 2018;64:645-55.

32. Fahim MA, Hayen AD, Horvath AR, et al. Biological variation of high sensitivity cardiac troponin-T in stable dialysis patients: implications for clinical practice. Clin Chem Lab Med 2015;53:715-22.

33. Aakre KM, Roraas T, Petersen PH, et al. Weekly and 90-minute biological variations in cardiac troponin $\mathrm{T}$ and cardiac troponin $\mathrm{I}$ in hemodialysis patients and healthy controls. Clin Chem 2014;60:838-47.

34. Mbagaya W, Luvai A, Lopez B. Biological variation of cardiac troponin in stable haemodialysis patients. Ann Clin Biochem 2015;52:562-8.

35. Corte Z, Garcia C, Venta R. Biological variation of cardiac troponin T in patients with end-stage renal disease and in healthy individuals. Ann Clin Biochem 2015;52:53-60.

36. van der Linden N, Hilderink JM, Cornelis T, et al. Twenty-four-hour biological variation profiles of cardiac troponin I in individuals with or without chronic kidney disease. Clin Chem 2017;63:1655-6.

37. Apple FS, Sandoval Y, Jaffe AS, et al. Cardiac troponin assays: guide to understanding analytical characteristics and their impact on clinical care. Clin Chem 2017;63:73-81.

38. National Institute for Health and Care Excellence. Myocardial infarction (acute): early rule out using high-sensitivity troponin tests (Elecsys Troponin T high-sensitive, ARCHITECT STAT High Sensitive Troponin-I and AccuTnI+3 assays). 2014;DG15: https://www.nice.org.uk/guidance/dg15. 
39. Klinkenberg LJ, van Dijk JW, Tan FE, et al. Circulating cardiac troponin T exhibits a diurnal rhythm. J Am Coll Cardiol 2014;63:1788-95.

40. Fournier S, Iten L, Marques-Vidal P, et al. Circadian rhythm of blood cardiac troponin T concentration. Clin Res Cardiol 2017;106:1026-32.

41. Egger M, Dieplinger B, Mueller T. One-year in vitro stability of cardiac troponins and galectin-3 in different sample types. Clin Chim Acta 2018;476:117-22.

42. Wu AH, Lu QA, Todd J, et al. Short- and long-term biological variation in cardiac troponin I measured with a high-sensitivity assay: implications for clinical practice. Clin Chem 2009;55:52-8.

43. Frankenstein $\mathrm{L}, \mathrm{Wu} \mathrm{AH}$, Hallermayer $\mathrm{K}$, et al. Biological variation and reference change value of high-sensitivity troponin $\mathrm{T}$ in healthy individuals during short and intermediate follow-up periods. Clin Chem 2011;57:1068-71. 
Table 1. Characteristics of the study population. Corresponding values for continuous data are shown as median (range). Laboratory median data (serum creatinine, estimated GFR and plasma cardiac troponin concentrations) represent the median of all values obtained during the four week study period.

\section{$\mathrm{n}$}

Age, years

$71(50-80)$

M:F

$10: 10$

Caucasian (n) 20

Height, cm $170.5(154-194)$

Weight, $\mathrm{kg}$

$79.5(47.1-118.1)$

Body surface area, $\mathrm{m}^{2}$

Body mass index (median of the individual BMI values)

$28.2(19.6-40.9)$

Serum creatinine, $\mu \mathrm{mol} / \mathrm{L}$

Estimated GFR calculated using MDRD equation, $\mathrm{mL} / \mathrm{min} / 1.73 \mathrm{~m}^{2}$

Plasma cardiac troponin I

Median concentration, $\mathrm{ng} / \mathrm{L}$

Minimum concentration, $\mathrm{ng} / \mathrm{L}$

Maximum concentration, $\mathrm{ng} / \mathrm{L}$

Number of patients with median $\mathrm{cTnI}>$ appropriate $99^{\text {th }}$ percentile

$0 / 18$

Plasma cardiac troponin $\mathrm{T}$

Median concentration, $\mathrm{ng} / \mathrm{L}$

Minimum concentration, ng/L

Maximum concentration, $\mathrm{ng} / \mathrm{L}$

Number of patients with median cTnT concentration >99th 7/18 percentile 
Table 2. Summary of the components of variation of cardiac troponin I and T.

Components of Variation

Analytical variation $\left(\mathrm{CV}_{\mathrm{A}}\right)$

Within-subject variation $\left(\mathrm{CV}_{\mathrm{I}}\right)$

Between-subject variation $\left(\mathrm{CV}_{\mathrm{G}}\right)$

Total variation $\left(\mathrm{CV}_{\mathrm{T}}\right)$

Positive log-normal RCV (rise)

Negative log-normal RCV (fall)

\section{Cardiac Troponin I}

$8.3 \%(7.0 \%, 10.2 \%)$

$15.0 \%(11.6 \%, 20.2 \%)$

$105.6 \%(72.0 \%, 210.8 \%)$

$17.2 \%$

$60 \%$

$-38 \%$

\section{Cardiac Troponin T}

$3.1 \%(2.6 \%, 3.8 \%)$

$7.4 \%(5.9 \%, 9.8 \%)$

$78.4 \%(55.6 \%, 139.4 \%)$

$8.0 \%$

$25 \%$

$-20 \%$

Values are $\%(95 \% \mathrm{CI}) ; \mathrm{CI}=$ confidence interval; $\mathrm{CV}_{\mathrm{A}}=$ analytical variation; $\mathrm{CV}_{\mathrm{G}}=$ between-subject biological variation; $\mathrm{CV}_{\mathrm{I}}=$ within-subject biological variation; $\mathrm{RCV}=$ reference change value. 
Fig. 1. Plots depicting the biological variation of cardiac troponins $T$ (A) and $I$ (B) in 18 patients (minus exclusions) with moderate CKD sampled over a period of 4 weeks. Diamonds represent the individual patient medians with the filled circles representing individual minimum and maximum values. Males and females are depicted by blue and red diamonds respectively. For cardiac troponin $\mathrm{T}$, the horizontal dashed line represents the upper $99^{\text {th }}$ percentile of a healthy reference population. In the case of cardiac troponin I, the horizontal dashed line represents the $99^{\text {th }}$ percentile for females $(15.6 \mathrm{ng} / \mathrm{L})$, with males having a higher $99^{\text {th }}$ percentile (34.2 ng/L, not shown). 
A

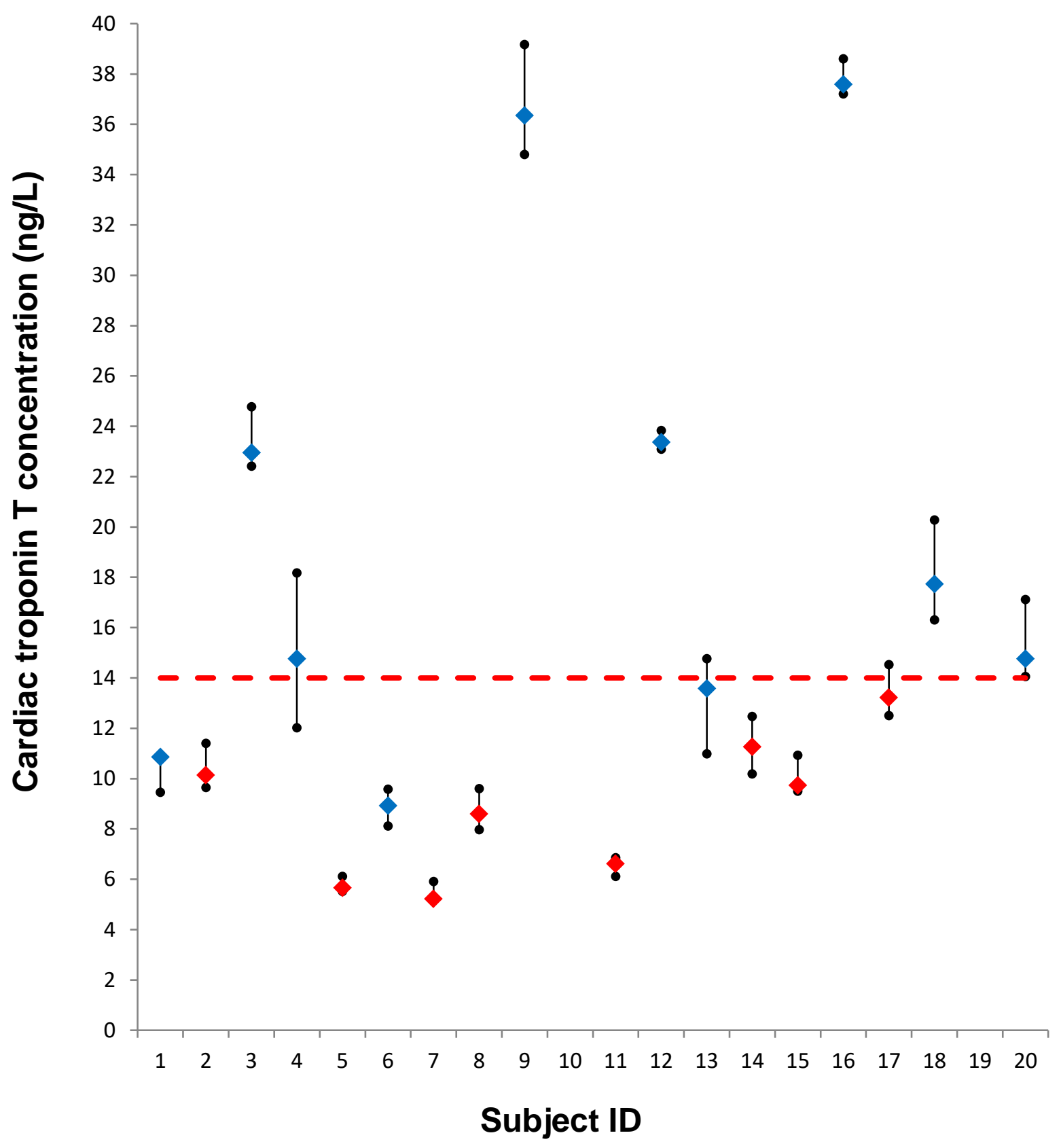


B

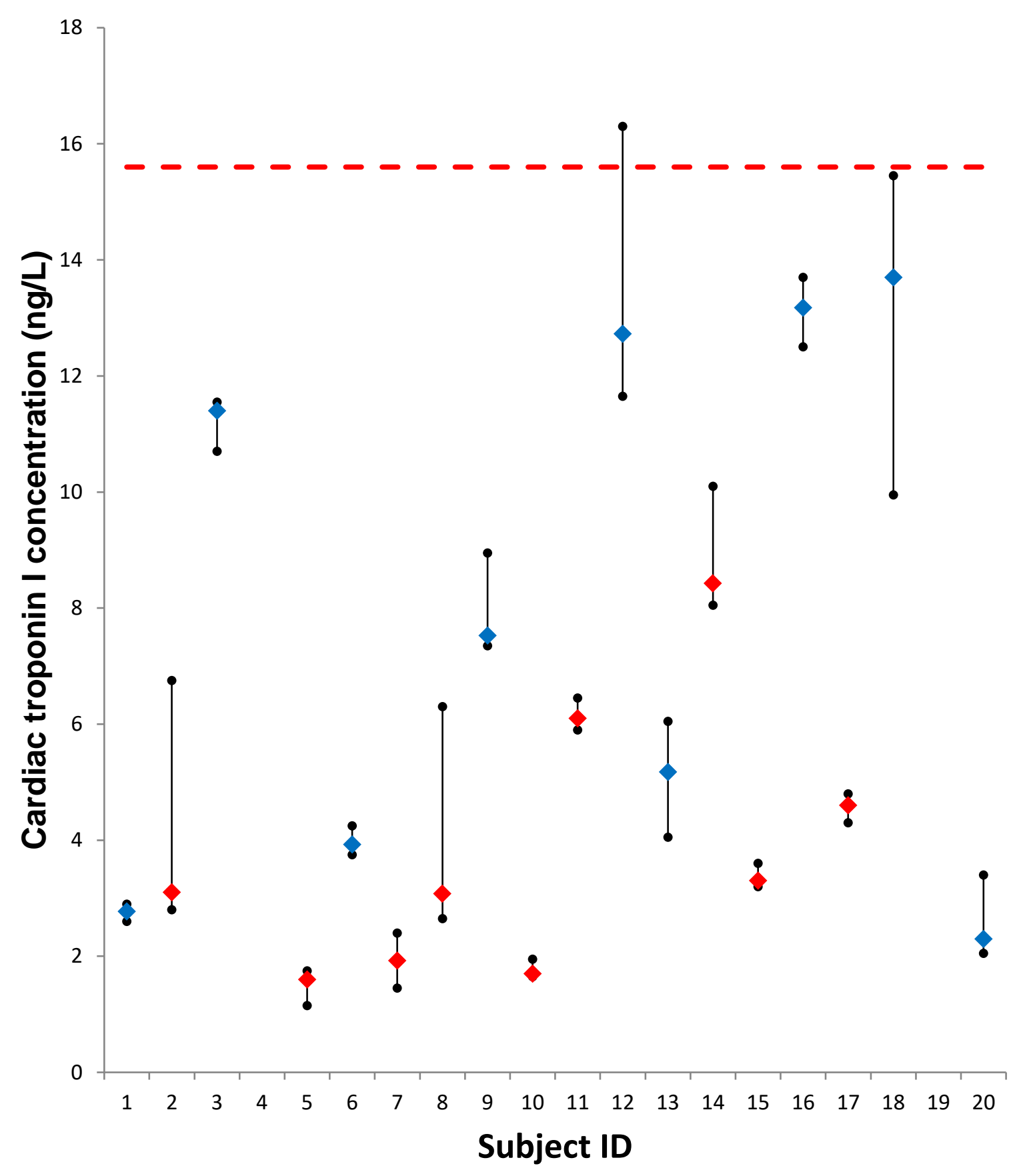




\section{Supplementary File}

Supplementary Table S1. Outliers are represented in this table by patient ID number (e.g. B10551) and week of sample (e.g. week 1=A, week $2=\mathrm{B}$ etc).

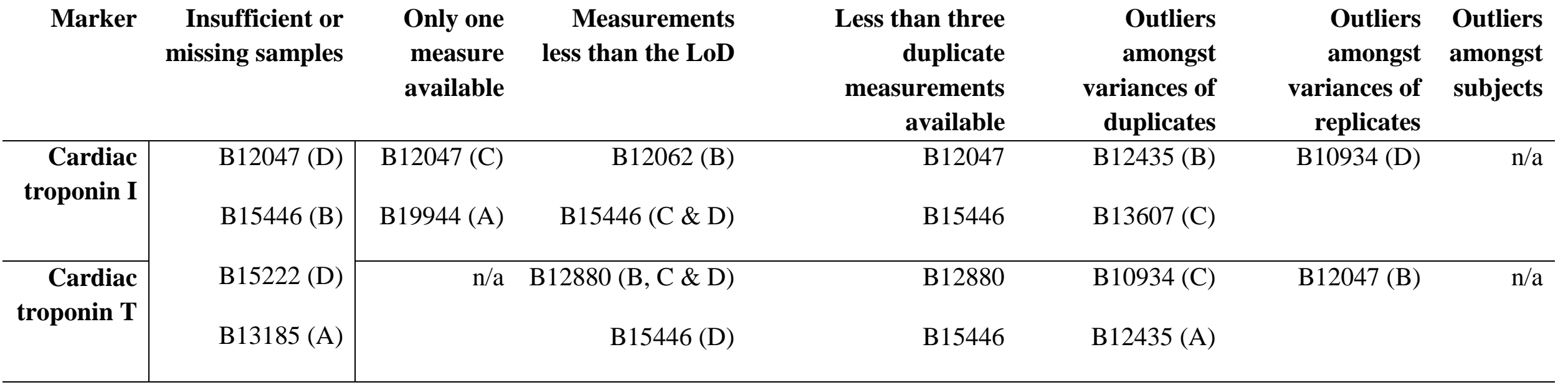

LoD, limit of detection 